\title{
Purine Catabolism in Polymorphonuclear Neutrophils

\author{
Phorbol Myristate Acetate-induced Accumulation of Adenosine Owing \\ to Inactivation of Extracellularly Released Adenosine Deaminase
}

\author{
Geert van Waeg and Georges Van den Berghe \\ Laboratory of Physiological Chemistry, International Institute of Cellular and Molecular Pathology, B-1200, Brussels, Belgium
}

\begin{abstract}
Since physiological concentrations $(0.1-1 \mu \mathrm{M})$ of adenosine influence the functions of human polymorphonuclear neutrophils (PMNs), we investigated the metabolism of adenosine in suspensions of stimulated and unstimulated PMNs. Stimulation with phorbol myristate acetate (PMA, $1 \mu M$ ), but not by zymosan (0.5 mg/ml) or $\boldsymbol{N}$-formyl-methionyl-leucyl-phenylalanine (fMLP, $1 \mu \mathrm{M})$, provoked an accumulation of endogenous adenosine at a rate of $2.3 \pm 1.0 \mathrm{amol} / \mathrm{cell}$ per minute. A similar accumulation was observed with both unstimulated and stimulated PMNs after the addition of deoxycoformycin (dCF, 1-100 $\mu \mathrm{M})$, an inhibitor of adenosine deaminase. Exogenous adenosine $(10 \mu \mathrm{M})$ was deaminated at a rate of $9.8 \pm 3.7 \mathrm{amol} /$ cell per minute in control or zymosan or fMLP-stimulated PMN suspensions. This deamination was nearly completely suppressed when the PMNs had been stimulated with PMA. In contrast, the activity of adenosine deaminase in PMN lysates (231 \pm 72 amol/cell per minute) was not modified by PMA stimulation. $\alpha, \beta$-Methyleneadenosine 5'-diphosphate (AMPCP, $2.5 \mathrm{mM}$ ), an inhibitor of membranous ecto-5'-nucleotidase, profoundly inhibited endogenous adenosine accumulation under all conditions. PMA stimulation also provoked an inactivation of extracellular adenosine deaminase, purine nucleoside phosphorylase, and lactate dehydrogenase in PMN suspensions. We concluded that PMNs, even when not stimulated, continuously produce adenosine by dephosphorylation of extracellularly released adenylates; and that stimulation of PMNs by PMA causes adenosine accumulation owing to the inactivation of adenosine deaminase released by broken cells. (J. Clin. Invest. 1991. 87:305-312.) Key words: deoxycoformycin; nucleoside transport; 5'-nucleotidase(s); phagocytosis
\end{abstract}

\section{Introduction}

Addition of physiological $(0.1-1 \mu \mathrm{M})$ concentrations of adenosine to suspensions of human polymorphonuclear neutrophils (PMNs) influences their function. Superoxide anion production $(1,2)$ and aggregation (3) in response to various stim-

This work was published in part in abstract form in 1989 (Ann. Nutr. \& Metab. 33:229-230).

Address reprint requests to Dr. G. Van den Berghe, Laboratory of Physiological Chemistry, I. C. P. 75.39, Avenue Hippocrate 75, B-1 200 Brussels, Belgium.

Dr. Waeg's present address is Research and Development, Terumo Europe, B-3001, Heverlee, Belgium.

Received for publication 25 January 1990 and in revised form 15 June 1990

J. Clin. Invest.

(C) The American Society for Clinical Investigation, Inc.

0021-9738/91/01/0305/08 \$2.00

Volume 87, January 1991, 305-312 uli are inhibited, whereas chemotaxis is enhanced (4). These effects have been shown to involve adenosine receptors (4-7). Adenosine might thus be a physiologically important inhibitor of PMN-mediated cell injury $(4,8)$.

Adenosine is an intermediate of the catabolism of the adenine nucleotides. These are maintained in equilibrium by adenylate kinase, and ultimately broken down to hypoxanthine in most human cell types, including PMNs (Fig. 1). Adenine nucleotide catabolism can occur by two pathways that differ in two metabolic steps. The branchpoint is at AMP, which can either be dephosphorylated to adenosine by 5 '-nucleotidase(s), or deaminated to inosine $5^{\prime}$-monophosphate (IMP) ${ }^{1}$ by AMP deaminase. The adenosine formed along the right-hand pathway does not necessarily accumulate, as it can be either rephosphorylated to AMP by adenosine kinase (9), or deaminated to inosine by adenosine deaminase (ADA). IMP, formed along the left-hand pathway, is dephosphorylated to inosine, most likely by a different 5 -nucleotidase than that which acts on AMP $(10,11)$. Purine nucleoside phosphorylase (PNP) splits inosine into hypoxanthine and ribose 1-P.

Phagocytic stimuli are known to induce ATP breakdown in PMNs (12-14). Although the presence of adenosine in suspensions of human PMNs has been documented $(1,15)$, little additional information is available with respect to production, uptake, and degradation, and the resulting accumulation of adenosine by these cells, at rest and upon stimulation. In this work, the catabolic pathway of the adenine nucleotides and the accumulation of adenosine have been studied in detail in suspensions of human PMNs, incubated under resting conditions and in the presence of the following stimuli: zymosan, a particulate extract from yeast; $N$-formyl-methionyl-leucyl-phenylalanine (fMLP), a chemotactic peptide; and phorbol myristate acetate (PMA), a stimulator of protein kinase C. Our results show that stimulation of PMNs by PMA provokes an extracellular accumulation of adenosine due to inactivation of ADA released by the cells. Adenosine formed by the PMNs themselves might thus inhibit their capacity to injure cells.

\section{Methods}

Materials. Nucleotides, nucleosides, bases, and auxiliary enzymes were from Boehringer GmbH (Mannheim, FRG). $\left[8-{ }^{14} \mathrm{C}\right] \mathrm{ATP}(\mathrm{sp}$ act $=58$ $\mathrm{Ci} / \mathrm{mol}),\left[8^{14} \mathrm{C}\right] \mathrm{AMP}(55 \mathrm{Ci} / \mathrm{mol}),\left[8-{ }^{14} \mathrm{C}\right]$ adenosine $(59 \mathrm{Ci} / \mathrm{mol})$, and $\left[8-{ }^{14} \mathrm{C}\right]$ inosine $(57 \mathrm{Ci} / \mathrm{mol})$ were from the Radiochemical Centre, Ltd., Amersham, Bucks., UK). Deoxycoformycin (dCF) was from WarnerLambert Co., Ann Arbor, MI. 5-lodotubercidin was from Research Biochemicals, Inc., (Natick, MA). Soluflazin was a gift from $H$. van

1. Abbreviations used in this paper: ADA, adenosine deaminase (EC 3.5.4.4.); AMPCP, $\alpha, \beta$-methyleneadenosine 5 -diphosphate; BDD, bromododecane; dCF, deoxycoformycin; IMP, inosine $5^{\prime}$-monophosphate; LDH, lactate dehydrogenase (EC 1.1.1.27); PCA, perchloric acid; PEI, polyethyleneimine; PNP, purine nucleoside phosphorylase (EC 2.4.2.1). 


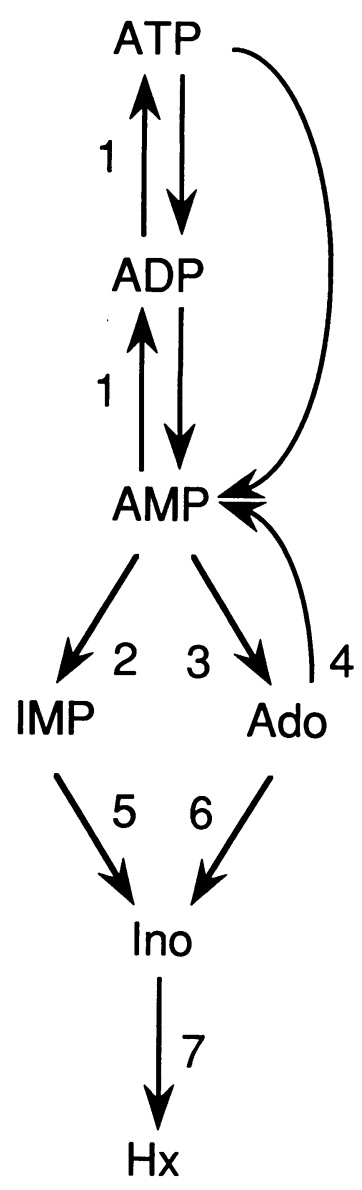

Figure 1. Pathways of ATP catabolism. Numbers refer to: (1) adenylate kinase; (2) AMP deaminase; (3) (AMP-) 5'-nucleotidase; (4) adenosine kinase; (5) (IMP-GMP-) 5'-nucleotidase; (6) adenosine deaminase; (7) purine nucleoside phosphorylase. Ado, adenosine; Ino, inosine; Hx, hypoxanthine.

Belle (Janssen Research, Beerse, Belgium). Bromododecane (BDD) was from Janssen Chimica, Beerse, Belgium, Dextran T-500 and Sephadex G-25 (medium grade) from Pharmacia Inc., Uppsala, Sweden. All other chemicals were analytical grade from Sigma Chemical Co., St. Louis, MO.

KRB contained, unless given otherwise, (in $\mathrm{mM}$ ): $\mathrm{NaCl} 118, \mathrm{KCl} 5$, $\mathrm{KH}_{2} \mathrm{PO}_{4} 1.2, \mathrm{MgSO}_{4} 1.2, \mathrm{NaHCO}_{3} 25, \mathrm{CaCl}_{2} 2.5$; was always supplemented with glucose $5 \mathrm{mM}$; and was flushed with $\mathrm{O}_{2} / \mathrm{CO}_{2}$ (95\%:5\%).

PMA and $\mathrm{MLP}$ were dissolved in DMSO. To control incubations, $0.1 \%$ DMSO was added. No difference in the parameters monitored was observed between 0 and $1 \%$ DMSO added. All other solutions were prepared in distilled water. Zymosan was boiled for $1 \mathrm{~h}$ in $\mathrm{NaCl} 0.9 \%$ at $10 \mathrm{mg} / \mathrm{ml}$, washed twice, opsonized $30 \mathrm{~min}$ at $37^{\circ} \mathrm{C}$ in fresh plasma (mixed from three donors), washed twice, and resuspended in KRB. Unless otherwise stated, the following final concentrations were used: PMA $1 \mu \mathrm{M}$, fMLP $1 \mu \mathrm{M}$, zymosan $0.5 \mathrm{mg} / \mathrm{ml}$, and 2-deoxyglucose $10 \mathrm{mM}$.

Donors. Most experiments were performed with PMNs isolated from blood from patients with hemochromatosis. However, every single experiment reported in this paper has been performed at least once with PMNs obtained from healthy volunteers, and no qualitative or quantitative difference was observed in any of the parameters described in this paper.

Isolation of neutrophils. Packed blood cells (200-250 ml) were obtained by centrifugation of peripheral venous blood at $3,000 \mathrm{~g}$ for 10 $\mathrm{min}$. The cells were mixed with an equal volume of $\mathrm{KRB}$, in which $\mathrm{CaCl}_{2}$ was omitted and $3 \%(\mathrm{wt} / \mathrm{vol})$ dextran $\mathrm{T}-500$ was added. They were allowed to sediment during $1 \mathrm{~h}$ at room temperature. The leukocyte-rich supernate was transferred into a $250-\mathrm{ml}$ centrifugation tube (Dow-Corning Corp., Midland, MI) and centrifuged $5 \mathrm{~min}$ at $200 \mathrm{~g}$. The supernate was discarded and the cells resuspended in $1 \mathrm{ml} \mathrm{KRB}$ by repeated aspiration in an automatic pipettip. A hemolytic shock was performed by adding $42 \mathrm{ml}$ bidistilled $\mathrm{H}_{2} \mathrm{O}$, followed after $20 \mathrm{~s}$ by 14 $\mathrm{ml} \mathrm{NaCl} 3.5 \%$. After centrifugation $(5 \mathrm{~min}, 200 \mathrm{~g}$ ), the cells were washed three times with $20 \mathrm{ml} \mathrm{KRB}$ and centrifuged $5 \mathrm{~min}$ at 150,100 , and $60 \mathrm{~g}$, respectively. The cells obtained in this way were typically $>93 \%$ PMNs, and viability, as judged by trypan blue exclusion, was $>96 \%$.

Incubations. Cell suspensions, containing between 3 and $60 \cdot 10^{6}$ cells $/ \mathrm{ml}$, were incubated in $\mathrm{KRB}$ at $25^{\circ} \mathrm{C}$, in siliconized glass liquid scintillation vials, in a shaking water bath. Care was taken to provide sufficient flushing with $95 \% \mathrm{O}_{2}, 5 \% \mathrm{CO}_{2}$. For determinations of metabolites in the total cell suspension, $400 \mu$ l total incubation medium was pipetted into $100 \mu \mathrm{l}$ ice-cold $10 \%$ perchloric acid (PCA) at the times indicated. After centrifugation at $10,000 \mathrm{~g}$ for $3 \mathrm{~min}, 460 \mu \mathrm{l}$ of the supernate was transferred into a conical Eppendorf tube and neutralized with $100 \mu \mathrm{l}$ of a neutralization mixture containing $\mathrm{KOH} 1.08$ $\mathrm{M}, \mathrm{KHCO}_{3} 1.08 \mathrm{M}$, and bromthymol blue $77 \mu \mathrm{M}$.

When cells and extracellular medium had to be separated, the method of Cornell (16) was used, adapted as follows. $400 \mu 1$ BDD was layered on top of $200 \mu \mathrm{l}$ PCA $10 \%$ in a conical Eppendorf tube. $500 \mu \mathrm{l}$ PMN suspension was carefully layered on top of the BDD. Care was taken to avoid having the first drop penetrate the BDD layer and consequently to come in contact with the PCA. The tubes were centrifuged $1 \mathrm{~min}$ at $10,000 \mathrm{~g}$. Up to $400 \mu \mathrm{l}$ of supernate could be recovered by using a $100-\mu \mathrm{l}$ automatic pipette, and frozen in $\mathrm{CO}_{2}$ /acetone for enzyme analysis and/or added to PCA (4 vol of supernate to 1 vol of PCA $10 \%$ ) and neutralized after $30 \mathrm{~min}$ by adding 1 vol of the mixture given above. After carefully removing the remaining supernate, as well as the major part of the BDD, the precipitate was resuspended with a plastic rod, recentrifuged ( $3 \mathrm{~min}, 10,000 \mathrm{~g}$ ), and $140 \mu \mathrm{l}$ of the PCA layer was transferred to another Eppendorf tube containing $100 \mu \mathrm{l}$ of the neutralization mixture given above.

For determination of the catabolism of adenosine in the total cell suspension, $\left[8-{ }^{14} \mathrm{C}\right]$ adenosine $(10 \mu \mathrm{M})$ was added to the incubation medium. At appropriate time intervals, aliquots of the total cell suspension were pipetted into ice-cold PCA, centrifuged, and neutralized as described above. $10 \mu \mathrm{l}$ of the neutralized supernate was spotted on polyethyleneimine (PEI) cellulose TLC plates on which $50 \mathrm{nmol}$ of carrier adenosine, inosine, and hypoxanthine had been applied. After development in butan-1-ol/acetic acid/water (2:1:1, vol/vol), radioactivity was measured in the spots corresponding to the carriers.

For determination of adenosine uptake, $\left[8-{ }^{14} \mathrm{C}\right]$ adenosine $(10 \mu \mathrm{M})$, was added to cell suspensions that had been preincubated for $10 \mathrm{~min}$ in the presence of $100 \mu \mathrm{M} \mathrm{dCF}$ to inhibit adenosine deaminase. At appropriate time intervals, aliquots of the total cell suspension were pipetted into ice-cold PCA and processed as described in the previous paragraph, except that $50 \mathrm{nmol}$ carrier AMP, ADP, and ATP were also applied on the PEI cellulose TLC plates. For determination of adenosine uptake, the radioactivity appearing in the nucleotide spots was measured.

Enzyme activities on the extracellular medium were determined after centrifugation of the cells. For determination of enzyme activities on cell lysates, total cell suspensions were collected in $10 \mathrm{vol}$ of distilled water immediately before freezing in acetone $/ \mathrm{CO}_{2}$. Thereafter they were thawed and sonicated three times for $10 \mathrm{~s}$ on ice.

Quantitation of purine nucleotides, nucleosides, and bases. Purine metabolites were analyzed as previously (17) using HPLC on PartisilSAX (nucleotides) and $\mu$-Bondapack (nucleosides and bases), both from Whatman, Maidstone, Kent, UK.

Measurements of enzyme activities. The activity of ADA in extracellular medium and cell lysates was measured with $10 \mu \mathrm{M}\left[8-{ }^{14} \mathrm{C}\right]-$ adenosine. At three appropriate time intervals, $10 \mu \mathrm{l}$ of the incubation medium was spotted on PEI cellulose TLC plates on which $50 \mathrm{nmol}$ of adenosine, inosine, and hypoxanthine had been applied. After development in butan-1-ol/acetic acid/water (2:1:1, vol/vol), the enzymic activity was calculated from the sum of the radioactivity appearing in inosine and hypoxanthine. PNP was assayed by the radiochemical method described by Vincent et al. (18), except that the PEI plates were developed as above. The activity of lactate dehydrogenase (LDH) was measured according to Vassault (19).

For the measurement of adenosine kinase, suspensions of freshly 
purified PMNs $\left(20 \cdot 10^{6}\right.$ cells $\left./ \mathrm{ml}\right)$ were sonicated three times $10 \mathrm{~s}$ on ice, centrifuged $3 \mathrm{~min}$ at $10,000 \mathrm{~g}$, and the supernate was filtered on Sephadex G-25. The protein-rich fraction was preincubated $15 \mathrm{~min}$ with dCF $100 \mu \mathrm{M}$. The assay medium, essentially adapted from Arch and Newsholme (20), contained (final concentrations in $\mathrm{mM}$ ) Tris 50, $\mathrm{KCl} \mathrm{100,} \mathrm{MgCl}_{2}$ 5, ATP 3, AMPCP 2.5, phosphocreatine 10, dCF 0.01, and creatine phosphokinase $(0.2 \mathrm{mg} / \mathrm{ml})$, adenylate kinase $(0.03$ $\mathrm{mg} / \mathrm{ml})$, and $\left[8-{ }^{14} \mathrm{C}\right]$ adenosine $(10 \mu \mathrm{M})$.

Statistics. All results of repeated experiments are given as means \pm SD. Significance was tested for by Students $t$ test, doubletailed.

\section{Results}

\section{Concentrations of purine nucleotides}

The intracellular concentration of ATP, measured after centrifugation of suspensions of freshly isolated human PMNs through a BDD layer, was $1,770 \pm 170 \mathrm{amol} / \mathrm{cell}(n=8)$ at the beginning of the incubations. In the absence of additions, the ATP concentration after 52 min of incubation was $8 \pm 6 \%$ lower than initially, corresponding to a mean loss of ATP of 2.7 amol/cell per minute (Fig. 2). Phagocytic stimuli accentuated the ATP decrease, which reached $\sim 20 \%$ following the addition of opsonized zymosan or fMLP, and $>60 \%$ following that of PMA. Addition of 2-deoxyglucose, an inhibitor of glycolysis, also induced a pronounced depletion of ATP. Under all experimental conditions, ADP (initial concentration $250 \pm 42 \mathrm{amol} / \mathrm{cell}$ ) and AMP (initially $35 \pm 6 \mathrm{amol} / \mathrm{cell}$ ) were barely modified over the duration of the incubations. The adenylate energy charge was $0.925 \pm 0.015$ throughout the control incubations. It was barely modified upon addition of zymosan or fMLP, and never decreased below 0.880 in the presence of PMA, or below 0.830 upon addition of 2-deoxyglucose. Transient increases in IMP were recorded upon addition of PMA, and, to a distinctly lesser extent, of 2-deoxyglucose, zymosan, and $\mathrm{fMLP}$ (Fig. 3). At its peak, $10 \mathrm{~min}$ after the addition of PMA, IMP accounted for $49 \pm 7 \%$ of the total adenylates lost so far. These results indicate deamination of AMP into IMP dur-

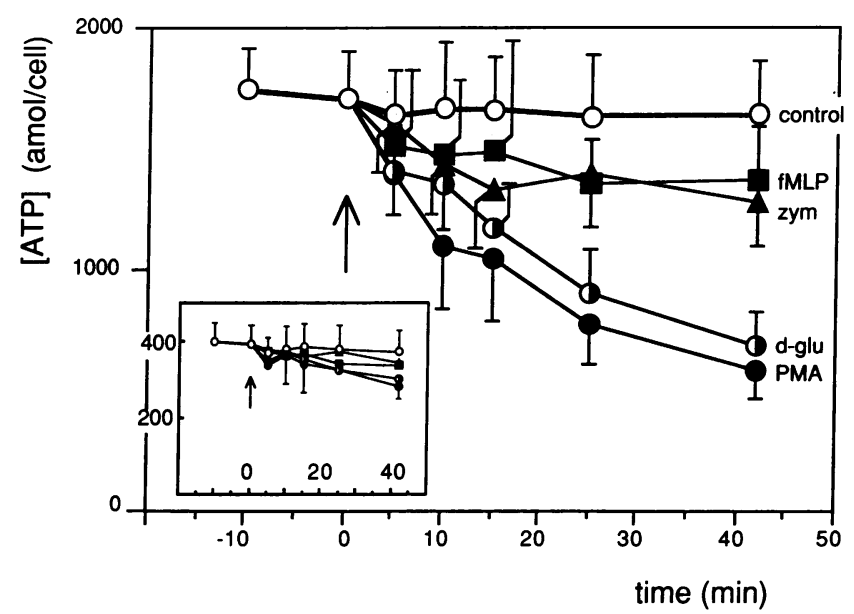

Figure 2. Intracellular concentration of ATP in freshly isolated human PMNs, incubated at $25^{\circ} \mathrm{C}$ in $\mathrm{KRB}$, under control conditions (O), or supplemented at zero time (arrow) with zymosan $(0.5 \mathrm{mg} / \mathrm{ml}$, $\Delta)$; fMLP (1 $\mu \mathrm{M}, \bullet)$; PMA (1 $\mu \mathrm{M}, \bullet)$; or 2-deoxyglucose (10 mM, $\bullet$, [d-glu]). Inset: Intracellular concentration of GTP, same experiments, same symbols. All concentrations are expressed in amol/cell, mean \pm SD of 7 (control) or 4 (other conditions) independent experiments conducted with PMNs from different donors.

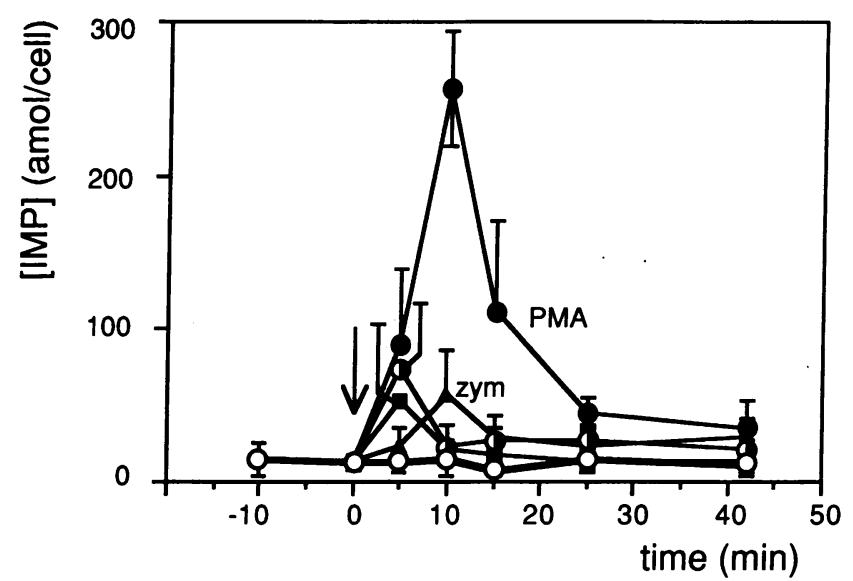

Figure 3. Intracellular concentration of IMP in freshly isolated human PMNs, incubated at $25^{\circ} \mathrm{C}$ in $\mathrm{KRB}$, under control conditions (O), or supplemented at zero time (arrow) with zymosan $(0.5 \mathrm{mg} / \mathrm{ml}$, $\Delta$ [zym]); fMLP (1 $\mu \mathrm{M}, \boldsymbol{\sigma})$; PMA (1 $\mu \mathrm{M}, \bullet)$; or 2-deoxyglucose (10 $\mathrm{mM}$, o). Results from 7 (control) or 4 (other conditions) independent experiments conducted with PMNs from different donors.

ing the catabolism of ATP induced by phagocytic stimuli and by 2 -deoxyglucose. The concentrations of the guanine nucleotides evolved similarly to those of the adenine nucleotides, but the relative decreases of guanosine 5 '-triphosphate (GTP) were less pronounced than those of ATP: GTP decreased from an initial concentration of $390 \pm 50 \mathrm{amol} / \mathrm{cell}$ to $280 \pm 30 \mathrm{amol} / \mathrm{cell}$ upon addition of PMA ( $n=4$; Fig. 2, inset).

Analysis of the incubation medium of the PMNs revealed that it contained $0.9 \pm 0.4 \mu \mathrm{M}$ ATP $(n=6)$ at the beginning of the incubations. This corresponds to $40 \pm 29$ amol of ATP for each cell present in the total suspension, or $2.3 \pm 1.7 \%$ of their total ATP (the suspensions in these experiments contained between 15 and $52 \cdot 10^{6}$ cells $\left./ \mathrm{ml}\right)$. The extracellular concentration of ADP was $0.7 \pm 0.4 \mu \mathrm{M}(n=6)$, equivalent to $30 \pm 28$ amol/cell. The extracellular concentration of AMP was $1.6 \pm 0.8 \mu \mathrm{M}(n=6)$, equivalent to $58 \pm 33 \mathrm{amol} / \mathrm{cell}$. Extracellular AMP accounted for $63 \pm 23 \%$ of total AMP in the PMN suspensions. No modifications of extracellular ATP, ADP, and AMP were observed over the duration of the experiments, either in control conditions or in the presence of the phagocytic stimuli or of 2-deoxyglucose. A negative correlation was observed between the fraction of total ATP (but not of ADP or AMP) located extracellularly (expressed as amol/cell or as \% of total ATP) and the cell concentration $(r=-0.878, P<0.05)$.

IMP was barely detectable in the extracellular medium at the beginning of the incubations. It rose slowly and approximately linearly to about $50 \mathrm{amol} / \mathrm{cell}$ after $\mathbf{4 2} \mathrm{min}$ in the different experimental conditions, including control (results not shown).

No significant changes in intracellular inorganic phosphate $\left(P_{i}\right)$ were observed upon addition of zymosan, fMLP, PMA, or 2-deoxyglucose to the PMNs (results not shown).

\section{Concentrations of adenosine, inosine, and hypoxanthine}

At the beginning of the incubations, the extracellular concentration of adenosine was $0.51 \pm 0.27 \mu \mathrm{M}(n=9)$, equivalent to $17 \pm 9 \mathrm{amol} / \mathrm{cell}$. This concentration did not change significantly over the duration of the experiments, either in control conditions or upon addition of fMLP, zymosan, or 2-deoxy- 


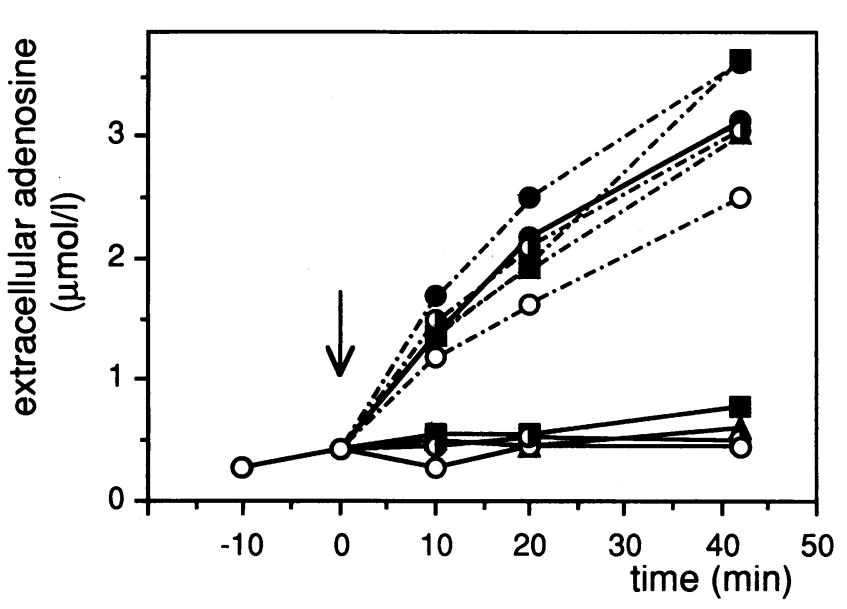

Figure 4. Extracellular concentration of adenosine in suspensions of freshly isolated human PMNs, incubated at $25^{\circ} \mathrm{C}$ in $\mathrm{KRB}$, under control conditions (O), or supplemented at zero time (arrow) with zymosan (0.5 mg/ml, $\Delta)$; fMLP (1 $\mu \mathrm{M}, \bullet)$; PMA (1 $\mu \mathrm{M}, \bullet)$; or 2-deoxyglucose $(10 \mathrm{mM}, \mathrm{o})$. Dashed lines represent the conditions in which, together with the additions mentioned above, $1 \mu \mathrm{M} \mathrm{dCF}$ was added. As relatively important intraindividual variations occur, the data of one experiment, representative for four to seven experiments combined in Table I, are given.

glucose (Fig. 4). In contrast, stimulation of the cells with PMA provoked a marked accumulation of adenosine, which proceeded at the rate of $2.3 \pm 1.0 \mathrm{amol} / \mathrm{cell}$ per minute $(n=9)$. At the end of the incubations, under control conditions and in the presence of zymosan or fMLP, the extracellular concentrations of inosine and hypoxanthine had increased nearly in parallel, and their sum accounted for the loss of purine nucleotides (not illustrated). Upon addition of PMA or 2-deoxyglucose, a severalfold higher increase was recorded for extracellular hypoxanthine, but the increase in extracellular inosine was similar to the one observed after stimulation with zymosan or fMLP. The sum of the extracellularly released catabolites, including PMA-induced adenosine, did not account for the loss of purine nucleotides.

Comparison of the concentrations of hypoxanthine in the incubation medium and in the total cell suspension showed that they were always the same, as was the case for adenosine. This indicates that these metabolites did not accumulate inside the cells. Inosine, on the other hand, was found to accumulate markedly in the total cell suspension but not in the extracellular medium under those conditions in which an important ATP breakdown was observed, namely in the presence of PMA or 2-deoxyglucose. This was confirmed by measuring inosine inside the cells after centrifugation through BDD. As shown in Fig. 5, inosine accumulated strongly intracellularly within $10 \mathrm{~min}$ after PMA and 2-deoxyglucose, and still > 70\% of total inosine was found intracellularly at the end of the incubation. This suggests that both intracellular phosphorolysis of inosine to hypoxanthine by PNP and transport of inosine out of the PMNs became rate-limiting when ATP breakdown was markedly accelerated. The sum of intra and extracellular purine catabolites accounted for the loss of ATP after PMA or 2-deoxyglucose.

\section{Metabolism of exogenous adenosine}

Adenosine, added at the concentration of $10 \mu \mathrm{M}$ to control PMN suspensions, was deaminated at the rate of $9.8 \pm 3.7$

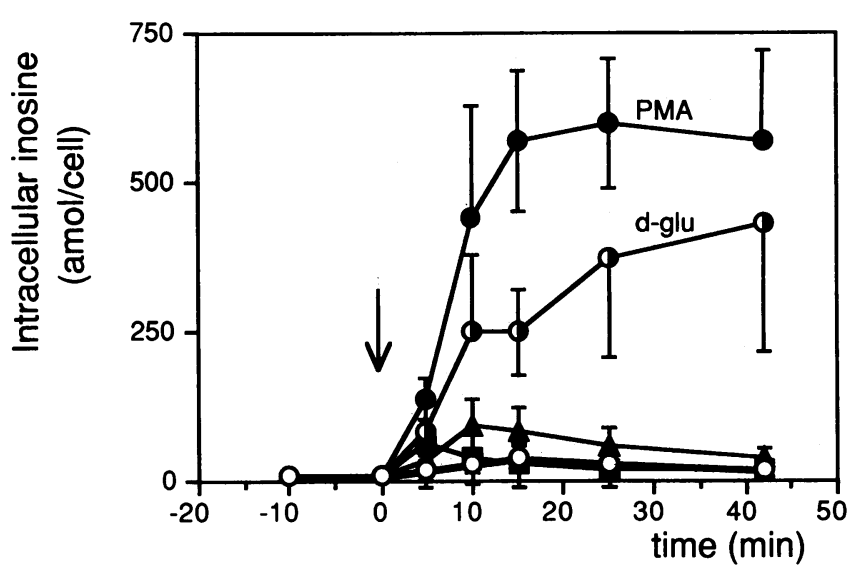

Figure 5. Intracellular concentration of inosine in freshly isolated human PMNs, incubated at $25^{\circ} \mathrm{C}$ in $\mathrm{KRB}$, under control conditions (O), or supplemented at zero time (arrow) with zymosan $(0.5 \mathrm{mg} / \mathrm{ml}$, $\Delta) ;$ fMLP (1 $\mu \mathrm{M}, \bullet)$; PMA (1 $\mu \mathrm{M}, \bullet)$; or 2-deoxyglucose (10 mM, o, [d-glu]). Results from seven (control and PMA) or four (other conditions) independent experiments conducted with PMNs from different donors.

amol/cell per minute $(n=10)$. This rate was not modified when the PMNs had been preincubated for 20 min with zymosan, fMLP, or 2-deoxyglucose. However, when the PMNs had been stimulated with PMA, the deamination of adenosine was nearly completely suppressed $(0.4 \pm 0.6 \mathrm{amol} / \mathrm{cell}$ per minute after $10 \mathrm{~min}, n=10$ ). Measurements of the activity of ADA in lysates of control PMNs revealed that it reached $231 \pm 72 \mathrm{amol} /$ cell per minute, which is about 25 -fold the rate of deamination measured in control PMN suspensions. The ADA activity in the lysates was not modified when the PMNs had been preincubated for 20 min with zymosan, fMLP, or 2-deoxyglucose before lysis. Neither was it modified when the cells had been stimulated with PMA 20 min before lysis. These data suggested that adenosine virtually does not penetrate inside the cells; that about $4 \%$ of the PMNs' ADA was located extracellularly; and that the addition of PMA to the cell suspension elevated adenosine by inhibiting or inactivating extracellular ADA.

The absence of appreciable penetration of adenosine inside the PMNs was confirmed by the observation that the addition of the inhibitors of nucleoside transport, dipyridamole and soluflazin (both at $10 \mu \mathrm{M}$ ), had no effect on the rate of degradation of exogenous adenosine $(10 \mu \mathrm{M})$ by intact PMN suspensions (results not shown).

Also in accordance with the absence of penetration of adenosine inside the PMNs was the observation that incubation of intact cells with $\left[8-{ }^{14} \mathrm{C}\right]$ adenosine $(10 \mu \mathrm{M})$, in the presence of $100 \mu \mathrm{M} \mathrm{dCF}$ to inhibit extracellular adenosine deamination (see below), did not result in any incorporation of the label into the cellular nucleotides. Nevertheless, an adenosine kinase activity of $26 \mu \mathrm{mol} / \mathrm{min}$ per gram of protein could be measured in PMN lysates.

\section{Influence of inhibition of adenosine deaminase}

To obtain further insights in the pathway of the degradation of the adenine nucleotides and in the mechanism of the accumulation of adenosine induced by PMA, experiments were performed with $\mathrm{dCF}$, a potent, tight binding inhibitor of ADA 
(21). In the presence of $1 \mu \mathrm{M} \mathrm{dCF}$ the deamination of $10 \mu \mathrm{M}$ $\left[{ }^{14} \mathrm{C}\right]$ adenosine by control PMN suspensions was completely inhibited $(<0.1 \mathrm{amol} / \mathrm{cell}$ per minute, $n=5)$. Addition of 1 $\mu \mathrm{M} \mathrm{dCF}$ to the cell suspension, incubated in control conditions or in the presence of zymosan, fMLP, or 2-deoxyglucose, induced a similar accumulation of adenosine as that observed upon addition of PMA in the absence of dCF (Fig. 4). Addition of dCF ( 1 or $100 \mu \mathrm{M}$ ) together with PMA did not significantly alter the adenosine accumulation as compared to dCF or PMA alone (Table I). These results indicate that adenosine is produced by the PMNs under control conditions but that the nucleoside does not accumulate owing to deamination by ADA; and that this production is not modified by fMLP, zymosan, 2-deoxyglucose, or PMA. The data also confirm the previous suggestion that the adenosine accumulation induced by PMA is caused by inhibition or inactivation of ADA.

ADA in lysates obtained from cells incubated for $0,7,15$, or $42 \mathrm{~min}$ with $1 \mu \mathrm{M} \mathrm{dCF}$ and washed three times before lysis was not inhibited. This indicates that, similar to adenosine and inosine, the inosine analogue $\mathrm{dCF}$ does not penetrate inside the PMNs. This result also confirms the previous suggestion that the accumulation of adenosine induced by PMA is caused by an effect on extracellular ADA. When PMN suspensions were incubated for $0,7,15$, or $42 \mathrm{~min}$ in the presence of 100 $\mu \mathrm{M} \mathrm{dCF}$, intracellular ADA assayed in lysates prepared after three washes of the cells was inhibited by $95 \%$ (results not shown). That the inhibition was already recorded at $0 \mathrm{~min}$ suggests that it was not caused by penetration of dCF inside the cells, but rather by an artifact as, e.g., adsorption of the inhibitor on the cell membrane, followed by release during the sonication procedure.

Neither the changes in endogenous adenosine or any of the other metabolites monitored, nor the disappearance rate of exogenous adenosine were affected in any of the conditions studied by adding 5-iodotubercidin, an inhibitor of adenosine kinase. This is most probably due to lack of transport of 5-iodotubercidin, a nucleoside analogue.

\section{Effect of inhibitors of ectonucleotidases}

The results described hitherto clearly indicated that the adenosine production, leading to adenosine accumulation in the presence of PMA or dCF, was due to extracellular degradation by ectonucleotidases of nucleotides released in the medium, rather than to intracellular catabolism of AMP by cytosolic

Table I. Extracellular Adenosine Concentration after 42 Min of Incubation

\begin{tabular}{lccc}
\hline & \multicolumn{3}{c}{$\mathrm{dCF} \mu \mathrm{M}$} \\
\cline { 2 - 4 } & 0 & 1 & 100 \\
\hline Control & $16 \pm 4(7)$ & $104 \pm 16(5)$ & $109 \pm 27(4)$ \\
PMA 1 $\mu \mathrm{M}$ & $122 \pm 33(7)$ & $145 \pm 60(5)$ & $142 \pm 33(4)$ \\
PMA 1 $\mu \mathrm{M}+$ AMPCP & & & \\
$2.5 \mathrm{mM}$ & $\mathrm{ND}$ & $\mathrm{ND}$ & $43 \pm 8(4)$ \\
\hline
\end{tabular}

Expressed in amol/cell (number of experiments). The values in italic are significantly different $(P<0.001)$ from each other and from all other values. The other values are not significantly different $(P$ $>0.01$ ) from each other.

ND, not determined.
5 -nucleotidase(s). To further substantiate this hypothesis, experiments were conducted with $\alpha, \beta$-methylene-adenosine $5^{\prime}$ diphosphate (AMPCP). This compound is a powerful inhibitor of ecto-5'-nucleotidase in various tissues (22). In suspensions of isolated PMNs, $2.5 \mathrm{mM}$ AMPCP inhibited the rate of degradation of $100 \mu \mathrm{M}$ exogenously added $\left[{ }^{14} \mathrm{C}\right] \mathrm{AMP}$ by $>90 \%$. We also found that AMPCP inhibited by $90 \%$ the degradation of $100 \mu \mathrm{M}\left[{ }^{14} \mathrm{C}\right] \mathrm{ATP}$, and induced a marked transient accumulation of radiolabeled ADP (results not shown), indicating inhibition of both ecto-ATPase(s) and ecto-ADPase(s). When $2.5 \mathrm{mM}$ AMPCP was added $15 \mathrm{~min}$ before PMA and dCF, the accumulation of adenosine was lowered more than fivefold (Table I). Concomitantly, AMPCP increased the concentrations of extracellular ATP and ADP, but not of AMP (results not shown). This indicates that the former nucleotides, rather than AMP, were released from the cells.

\section{Inactivation of extracellular adenosine deaminase, purine nucleoside phosphorylase, and lactate dehydrogenase}

To further substantiate the PMA-induced inactivation of extracellularly released ADA, its activity in the incubation medium was measured at various intervals over the whole duration of the experiments. As shown in Fig. $6 \mathrm{~A}$, about $3 \%$ of the total activity of ADA was in the extracellular medium at the beginning of the incubations. This proportion increased slowly and approximately linearly to around $9 \%$ after 52 min under control conditions as well as upon stimulation with zymosan or fMLP (not illustrated). Addition of PMA provoked a rapid inactivation of ADA, which persisted over the duration of the experiment. These findings prompted an evaluation of the influence of incubation and of the addition of PMA on PNP, another enzyme of purine catabolism, and on LDH, a classical index of cellular integrity. Fig. $6, B$ and $C$ show that the progressive accumulation of extracellular ADA recorded under control conditions was also found for PNP and LDH. These rates of accumulation were also not influenced by the addition of zymosan or fMLP (not illustrated). The overall release of the three enzymes over the duration of the experiments was $7 \pm 2 \%$, which is close to the loss of ATP in control conditions (Fig. 2). Similar to ADA, PNP and LDH were also inactivated by the addition of PMA, although inactivation of LDH was slower and not as pronounced as that of ADA or PNP.

\section{Discussion}

In accordance with others (12-14), we have observed that the phagocytic stimuli, zymosan, fMLP, and PMA, and the inhibitor of glycolysis, 2-deoxyglucose, induce catabolism of ATP in human PMNs. Our studies demonstrate that this intracellular catabolism proceeds, at least in part, via AMP deaminase, by the left-hand pathway depicted in Fig. 1. This leads to an intracellular accumulation of IMP and inosine, followed by the release of hypoxanthine in the extracellular medium (Fig. 7). In addition, we have found that suspensions of human PMNs continuously form adenosine at a fixed rate, both at rest and upon stimulation. This formation occurs extracellularly from adenine nucleotides released by the cells. It does not lead to accumulation of adenosine, unless the fraction of ADA which is released extracellularly is inhibited by dCF or inactivated by stimulation of the cells with PMA. The intra- and extracellular catabolism of adenine nucleotides in suspensions 


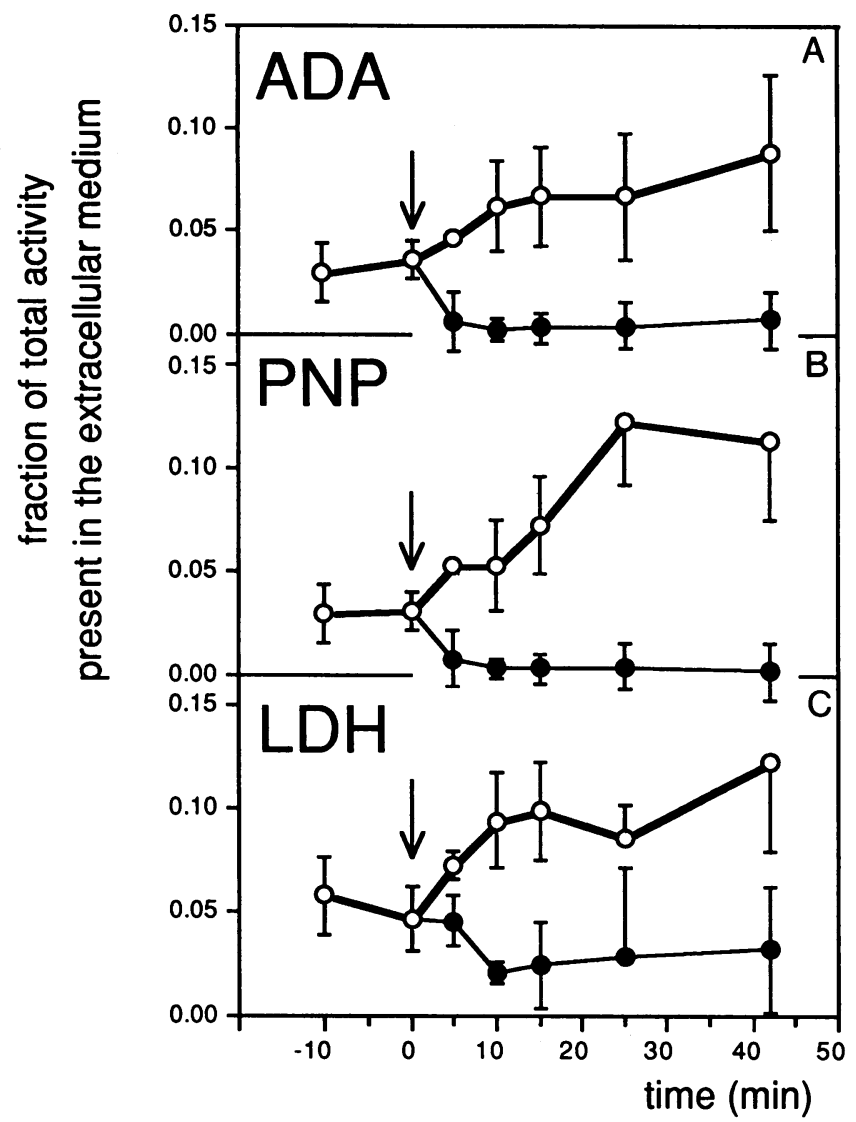

Figure 6. (A) Activity of adenosine deaminase in the extracellular medium of suspensions of freshly isolated human PMNs, incubated at $25^{\circ} \mathrm{C}$ in $\mathrm{KRB}$, under control conditions (o), or supplemented at zero time (arrow) with PMA $(1 \mu \mathrm{M}, \bullet)$. All activities are expressed as a fraction of the activity found in the total suspension after lysis of the cells, mean $\pm S D$ of three independent experiments conducted with PMNs from different donors. $(B)$ Activity of purine nucleoside phosphorylase, same experiments, same symbols. $(C)$ Activity of lactate dehydrogenase, same experiments, same symbols.

of human PMNs, the PMA-induced accumulation of adenosine, and information obtained with respect to the nucleoside transport system in these cells will be discussed separately.

\section{Intracellular catabolism of adenine nucleotides}

The decrease in ATP, induced in PMNs by stimuli of phagocytosis, has been explained by the extra amount of energy required for this process $(12,23$, and references therein). Similarly to others $(12,14)$, we have found that the decrease in ATP, whether induced by phagocytic stimuli or by inhibition of glycolysis by 2-deoxyglucose, was not accompanied by an elevation of ADP or AMP. This indicates that the increase in adenylate catabolism does not result from an elevation in substrate, but rather from a decreased inhibition or increased stimulation of one of the two enzymes that may catalyze the initial breakdown of AMP.

The accumulation of IMP, recorded upon addition of phagocytic stimuli or of 2-deoxyglucose, indicates that the catabolism of AMP proceeds at least in part by AMP deaminase by the left-hand pathway depicted in Figs. 1 and 7. AMP deaminase possesses complex kinetic properties (reviewed in 24) that vary from tissue to tissue, owing to the existence of iso-

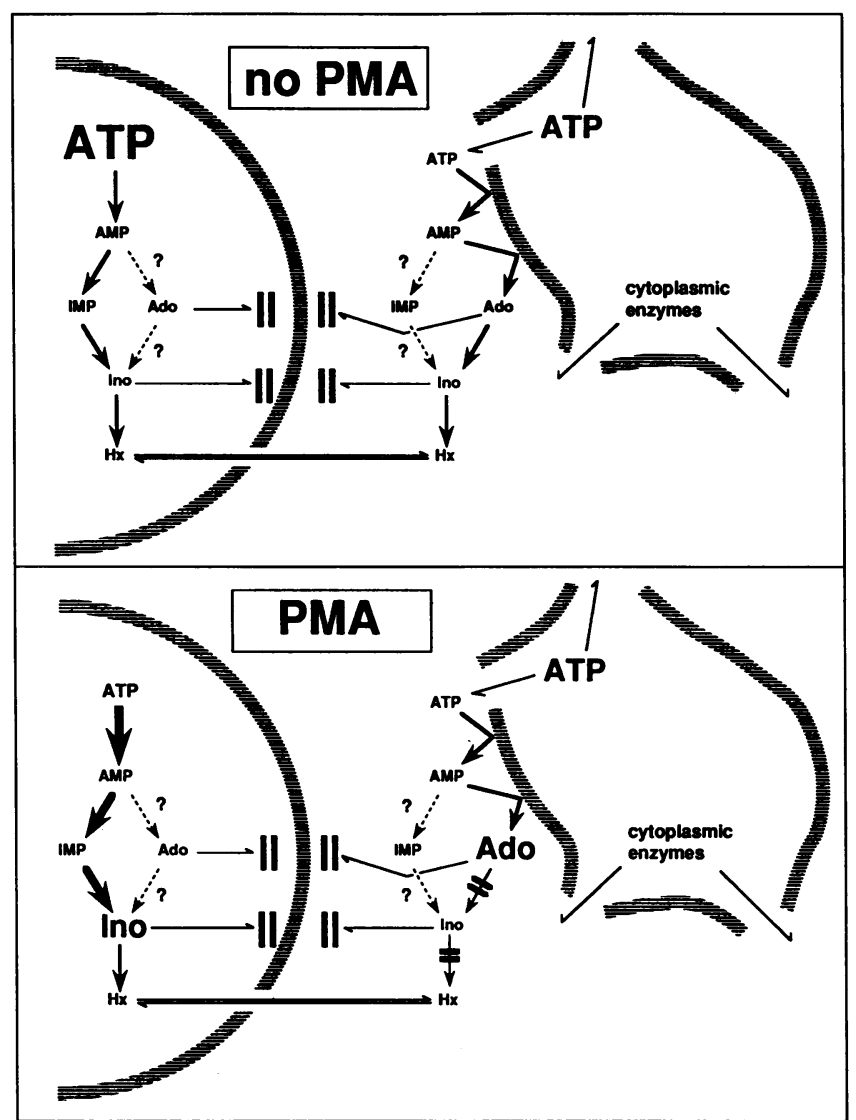

Figure 7. Schematic representation of adenylate catabolism in suspensions of human PMNs in control conditions (top) or upon stimulation with PMA (bottom) as concluded from the experiments described in this paper. Ado, adenosine; Ino, inosine; Hx, hypoxanthine.

zymes (25). ATP and ADP are stimulators, whereas GTP and $P_{i}$ are inhibitors of most of these isozymes. No significant changes were observed in intracellular $P_{i}$ or ADP. Whether the slight decrease in GTP $(-28 \%)$, which occurs in parallel with that of ATP, might be a sufficient explanation for the increase in the catabolism of the adenine nucleotide pool via AMP deaminase remains an open question. The observation that the pronounced, transient accumulation of IMP induced by PMA, which occurred intracellularly as expected, was followed by a similarly intracellular, lasting accumulation of inosine indicates that the dephosphorylation of IMP proceeds intracellularly, presumably by a specific IMP-GMP (guanosine monophosphate) 5'-nucleotidase $(11,26)$; that human PMNs have a very limited capacity to transport inosine; and that the activity of PNP becomes limiting at high rates of adenine nucleotide catabolism.

Whether catabolism of AMP also proceeds via its intracellular dephosphorylation to adenosine could not be determined, owing to the absence of penetration of the ADA inhibitor, dCF, inside the PMNs. The absence of penetration of both $\mathrm{dCF}$ and 5-iodotubercidin also precluded the determination of the existence of a futile recycling of adenosine, as demonstrated in isolated rat hepatocytes (9).

\section{Extracellular catabolism of adenine nucleotides}

In suspensions of resting PMNs and of cells incubated with fMLP, zymosan, or 2-deoxyglucose, the formation of adeno- 
sine could only be evidenced by addition of the ADA inhibitor dCF. In contrast, in cells stimulated with PMA, it was recorded in the absence of dCF. Several observations indicate that this formation of adenosine, its conversion into inosine in the absence of $\mathrm{dCF}$ or PMA, and its further catabolism into hypoxanthine occur extracellularly: (a) AMP was found in the incubation medium; $(b)$ formation of adenosine was markedly inhibited by AMPCP, an inhibitor of the membranous ecto-5'-nucleotidase; $(c)$ both ADA and PNP were found in the incubation medium; and $(d)$ adenosine accumulation required only $1 \mu \mathrm{M} \mathrm{dCF}$, a concentration which inhibited extracellular but not intracellular ADA.

Our results show that, similar to other cell types $(27,28)$, human PMNs release small amounts of nucleotides extracellularly. These nucleotides are most likely predominantly ATP and ADP, which are converted into AMP by the successive action of the ecto-ATPase(s) (29) and ecto-ADPase(s) (30) present on human PMN membranes. Indeed, addition of AMPCP, which we also found inhibitory of the latter ecto-enzymes, increased the extracellular concentration of ATP and ADP, but not that of AMP. The observation that ADA was found in medium from which the PMNs had been separated indicates, however, that ADA was released from the cells rather than present as an ecto-enzyme on their outer membrane. The extracellular release of adenine nucleotides is most likely due to unavoidable cell breakage during the incubations. This is indicated by the observation that, in control conditions, the loss of ATP and the release of ADA, PNP, and LDH occurred in parallel. In addition, the rate of adenosine formation in the presence of dCF or PMA in its turn corresponded to the loss of ATP in control conditions. The absence of modifications of the release of adenine nucleotides under the various experimental conditions indicates that these did not influence cell breakage. The constant rate of release of nucleotides also explains why the rates of accumulation of adenosine were similar when ADA was inhibited or inactivated. Cell breakage may nevertheless be a normal event in PMNs, and the accompanying release of adenosine may play a role in combination with ADA inactivation, as discussed in the next paragraph.

\section{Adenosine accumulation following stimulation with PMA}

As evidenced by the disappearance of the adenosine-deaminating activity from the incubation medium, the striking accumulation of adenosine observed upon stimulation of the PMNs with PMA results from inhibition of adenosine catabolism. The nearly complete loss of ADA activity in the incubation medium upon stimulation with PMA, together with the absence of effect of this medium on the ADA activity liberated upon lysis of the total suspension, argues against the generation of an ADA inhibitor by the PMNs upon PMA stimulation. Rather, it sustains the hypothesis that extracellular ADA is inactivated by intact, stimulated cells. This inactivation is most likely explained by the PMA-induced activation, by way of protein kinase $C$, of the bactericidal mechanisms of the PMNs. Why the other phagocytic stimuli, zymosan and fMLP, which also activate the bactericidal mechanisms, did not inactivate ADA, might be explained by several factors such as the more pronounced and longer lasting production of superoxide induced by PMA as compared with zymosan or fMLP $(31,32)$, or the more selective release, provoked by PMA, of the specific granules of PMNs (33). As yet it cannot be ruled out, however, that in certain pathophysiological con- ditions, in which the number of PMNs per unit volume of extracellular fluid is higher than in our experiments, stimulation of PMNs with natural stimuli might result in extracellular adenosine accumulation.

The inactivation of the three enzymes assayed (ADA, PNP, and LDH) suggests that the inactivation of extracellularly released enzymes by PMA-stimulated PMNs might be a general feature, but whether or not it is mediated by a common mechanism can only be found out by more directed experiments. The small difference in the shape of the inactivation curves between ADA and PNP on one side and LDH on the other side might indicate the operation of several inactivation mechanisms having distinct affinities for the different enzymes.

The significance of the inactivation of ADA and other enzymes by PMA is not immediately apparent. Since adenosine inhibits PMN-induced tissue injury (8), it may be one of the mechanisms whereby protein kinase $C$ exerts its general, protective role against cellular overstimulation (34). The PMAinduced accumulation of adenosine in PMN suspensions most likely accounts for the insensitivity to adenosine of the PMAinduced generation of superoxide $(1,35)$.

As evidenced by our data, extracellular LDH activity (and probably other enzyme activities as well) may not be used as a parameter of cell integrity of PMNs, at least not when stimulated with PMA.

\section{Nucleoside transport}

The marked rise of intracellular inosine recorded in association with high rates of ATP breakdown, without an equivalent increase in extracellular inosine, indicates that the nucleoside transporter is poorly efficient in the human PMN membrane. Other observations corroborate this conclusion: (a) the 25-fold lower rate of degradation of adenosine in suspensions of intact PMNs as compared with cell lysates; $(b)$ the absence of effect of inhibitors of adenosine transport on the rate of degradation of exogenous adenosine; $(c)$ the absence of incorporation of labeled adenosine into the nucleotides of intact PMNs, although adenosine kinase was found in PMN lysates; and $(d)$ the lack of penetration of $1 \mu \mathrm{M} \mathrm{dCF}$, a nucleoside analogue, inside the PMNs. To our knowledge, transport of nucleosides has not been investigated in detail in human PMNs. Cronstein et al. (1) have shown that $0.1 \mu \mathrm{M}$ adenosine, a 100-fold lower concentration than the one we used, disappeared from the incubation medium of PMNs and that this disappearance could be inhibited by dipyridamole. At up to $0.5 \mu \mathrm{M}$ adenosine little or no radioactivity was, however, found incorporated into adenine nucleotides (1). This suggests that low concentrations of adenosine may have been adsorbed on the cell membrane, similar to what we observed with dCF. Our results thus indicate that, in contrast to rabbit PMNs (36), but similar to sheep and dog erythrocytes (37), human PMNs may only have a poorly efficient, low capacity nucleoside transport system. That the formation of adenosine induced by 2-deoxyglucose in rat PMNs proceeded intracellularly and was accompanied by an increase in AMP (38) further indicates that important species differences exist with respect to the metabolic characteristics of PMNs.

\section{Acknowledgments}

This work was supported by grant 3.4539.87 of the Fund for Medical Scientific Research (Belgium) and by the Belgian State, Prime Minister's Office, Science Policy Programming. 
G. Van den Berghe is Director of Research of the Belgian National Fund for Scientific Research.

\section{References}

1. Cronstein, B. N., S. B. Kramer, G. Weissmann, and R. Hirschhorn. 1983. Adenosine: a physiological modulator of superoxide anion generation by human neutrophils. J. Exp. Med. 158:1160-1177.

2. Ward, P. A., T. W. Cunningham, K. K. McCulloch, S. H. Phan, J. Powell, and K. J. Johnson. 1988. Platelet enhancement of $\mathrm{O} \dot{\bar{z}}$-responses in stimulated human neutrophils. Identification of platelet factor as adenine nucleotide. Lab. Invest. 58:37-47.

3. Skubitz, K. M., N. W. Wickham, and D. E. Hammerschmidt. 1988. Endogenous and exogenous adenosine inhibit granulocyte aggregation without altering the associated rise in intracellular calcium concentration. Blood. 72:29-33.

4. Rose, F. R., R. Hirschhorn, G. Weissmann, and B. N. Cronstein. 1988. Adenosine promotes neutrophil chemotaxis. J. Exp. Med. 167:1186-1194.

5. Cronstein, B. N., E. D. Rosenstein, S. B. Kramer, G. Weissmann, and R. Hirschhorn. 1985. Adenosine; a physiologic modulator of superoxide anion generation by human neutrophils. Adenosine acts via an $A_{2}$ receptor on human neutrophils. J. Immunol. 135:13661371.

6. Schrier, D. J., and K. M. Imre. 1986. The effects of adenosine agonists on human neutrophil function. J. Immunol. 137:3284-3289.

7. Cronstein, B. N., S. B. Kramer, E. D. Rosenstein, H. M. Korchak, G. Weissmann, and R. Hirschhorn. 1988. Occupancy of adenosine receptors raises cyclic AMP alone and in synergy with occupancy of chemoattractant receptors and inhibits membrane depolarization. Biochem. J. 252:709-715.

8. Cronstein, B. N., R. I. Levin, J. Belanoff, G. Weissmann, and R. Hirschhorn. 1986. Adenosine: an endogenous inhibitor of neutrophilmediated injury to endothelial cells. J. Clin. Invest. 78:760-770.

9. Bontemps, F., G. Van den Berghe, and H. G. Hers. 1983. Evidence for a substrate cycle between AMP and adenosine in isolated hepatocytes. Proc. Natl. Acad. Sci. USA. 80:2829-2833.

10. Bontemps, F., G. Van den Berghe, and H. G. Hers. 1986. Pathways of adenine nucleotide catabolism in erythrocytes. J. Clin. Invest. 77:824-830.

11. Bontemps, F., G. Van den Berghe, and H. G. Hers. 1988. 5 -Nucleotidase activity in human erythrocytes. Identification of a purine 5'-nucleotidase stimulated by ATP and glycerate 2,3-bisphosphate. Biochem. J. 250:687-696.

12. Borregaard, N., and T. Herlin. 1982. Energy metabolism of human neutrophils during phagocytosis. J. Clin. Invest. 70:550-557.

13. Lane, T. A., and G. E. Lamkin. 1982. Defective energy metabolism in stored granulocytes. Transfusion (Phila.). 22:368-373.

14. Tauber, A. I., and M. F. Roberts. 1981. ${ }^{31}$ P NMR spectroscopy of phorbol-myristate-acetate stimulated polymorphonuclear human leukocytes. FEBS (Fed. Eur. Biochem. Soc.) Lett. 129:105-108.

15. Iannone, M. A., and G. Wolberg. 1987. Effects of adenosine on human neutrophil function and cyclic AMP content. In Topics and Perspectives in Adenosine Research. E. Gerlach and B. F. Becker, editors. Springer-Verlag, Berlin. 286-297.

16. Cornell, N. W. 1980. Rapid fractionation of cell suspensions with the use of brominated hydrocarbons. Anal. Biochem. 102:326331.

17. Bontemps, F., and G. Van den Berghe. 1989. Mechanism of adenosine triphosphate catabolism induced by deoxyadenosine and by nucleoside analogues in adenosine deaminase-inhibited human erythrocytes. Cancer Res. 49:4983-4989.

18. Vincent, M.-F., G. Van den Berghe, and H. G. Hers. 1984. Metabolism of hypoxanthine in isolated rat hepatocytes. Biochem. $J$. 222:145-155.

19. Vassault, A. 1983. Lactate dehydrogenase. UV method with pyruvate and NADH. In Methods of Enzymatic Analysis. Vol. 3. 3rd ed. H. U. Bergmeyer, editor. Verlag Chemie GmbH, Weinheim/ Bergst, FRG. 118-126.

20. Arch, J. R. S., and E. A. Newsholme. 1978. Activities and some properties of 5'-nucleotidase, adenosine kinase and adenosine deaminase in tissues from vertebrates and invertebrates in relation to the control of the concentration and the physiological role of adenosine. Biochem. J. 174:965-977.

21. Henderson, J. F., L. Brox, G. Zombor, D. Hunting, and C. A. Lomax. 1977. Specificity of adenosine deaminase inhibitors. Biochem. Pharmacol. 26:1967-1972.

22. Burger, R. M., and J. M. Lowenstein. 1975. 5'-Nucleotidase from smooth muscle of small intestine and from brain. Inhibition by nucleotides. Biochemistry. 14:2362-2366.

23. Sbarra, A. J., and M. L. Karnovsky. 1959. The biochemical basis of phagocytosis. I. Metabolic changes during the ingestion of particles by polymorphonuclear leukocytes. J. Biol. Chem. 234:13551362.

24. Zielke, C. L., and C. H. Suelter. 1971. Purine, purine nucleoside, and purine nucleotide aminohydrolases. The Enzymes. Vol. 4. 3rd ed. P. D. Boyer, editor. Academic Press, New York. 64-73.

25. Ogasawara, N., H. Goto, and T. Watanabe. 1975. Isozymes of rat AMP deaminase. Biochim. Biophys. Acta. 403:530-537.

26. Bontemps, F., M. F. Vincent, F. Van den Bergh, G. van Waeg, and G. Van den Berghe. 1989. Stimulation by glycerate 2,3-bisphosphate: a common property of cytosolic IMP-GMP 5'-nucleotidase in rat and human tissues. Biochim. Biophys. Acta. 997:131-134.

27. Van Schaftingen, E., L. Hue, and H. G. Hers. 1987. Extracellular metabolites in suspensions of isolated hepatocytes. Biochem. J. 248:517-521.

28. Kather, H. 1988. Purine accumulation in human fat cell suspensions. Evidence that human adipocytes release inosine and hypoxanthine rather than adenosine. J. Biol. Chem. 263:8803-8809.

29. Harlan, J., L. R. De Chatelet, D. B. Iverson, and C. E. McCall. 1977. Mg-dependent adenosine triphosphatase as a marker enzyme for the plasma membrane of human polymorphonuclear leukocytes. Infect. Immun. 15:436-443.

30. Smith, G. P., and T. J. Peters. 1981. Subcellular localization and properties of adenosine diphosphatase activity in human polymorphonuclear leukocytes. Biochim. Biophys. Acta. 673:234-242.

31. Sedgwick, J. B., R. F. Vrtis, M. F. Gourley, and W. W. Busse. 1988. Stimulus-dependent differences in superoxide anion generation by normal human eosinophils and neutrophils. J. Allergy Clin. Immunol. 81:876-883.

32. Burnham, D. N., S. R. Tyagi, D. J. Uhlinger, and J. D. Lambeth. 1989. Diacylglycerol generation and phosphoinositide turnover in human neutrophils: effects of particulate versus soluble stimuli. Arch. Biochem. Biophys. 269:345-353.

33. White, J. G., and R. D. Estensen. 1974. Selective labilization of specific granules in polymorphonuclear leukocytes by phorbol myristate acetate. Am. J. Pathol. 75:45-60.

34. Nishizuka, Y. 1986. Studies and perspectives of protein kinase C. Science (Wash. DC). 233:305-312.

35. McGarrity, S. T., A. H. Stephenson, and R. O. Webster. 1989. Regulation of human neutrophil functions by adenine nucleotides. $J$. Immunol. 142:1986-1994.

36. Taube, R. A., and R. D. Berlin. 1972. Membrane transport of nucleosides in rabbit polymorphonuclear leukocytes. Biochim. Biophys. Acta. 255:6-18.

37. Jarvis, S. M., J. R. Hammond, A. R. P. Paterson, and A. S. Clanachan. 1982. Species differences in nucleoside transport. Biochem. J. 208:83-88.

38. Newby, A. C., and C. A. Holmquist. 1981. Adenosine production inside rat polymorphonuclear leucocytes. Biochem. J. 200:399403. 